\title{
Highly Monodispersed PbS Quantum Dots for Outstanding Cascaded-Junction Solar Cells
}

Bo Hou, ${ }^{\dagger}$ Yuljae Cho, ${ }^{\dagger}$ Byung Sung Kim, ${ }^{\dagger}$ John Hong, ${ }^{\dagger}$ Jong Bae Park, ${ }^{\dagger}$ Se Jin Ahn, ${ }^{\S}$ Jung Inn Sohn, ${ }^{*}{ }^{\dagger}$ SeungNam Cha, ${ }^{* \dagger}$ and Jong Min Kim ${ }^{\|}$

${ }^{\dagger}$ Department of Engineering Science, University of Oxford, Parks Road, Oxford OX1 3PJ, U.K.

† Jeonju Centre, Korea Basic Science Institute, Jeonju, Jeollabuk-do 54907, Republic of Korea

${ }^{\S} \mathrm{New}$ and Renewable Energy Research Division, Photovoltaic Laboratory, Korea Institute of Energy Research (KIER), 152

Gajeong-ro, Yuseong-gu, Daejeon 305-343, Republic of Korea

"Department of Engineering, University of Cambridge, 9 JJ Thomson Avenue, Cambridge CB3 0FA, U.K.

Supporting Information

ABSTRACT: High-performance cascaded-junction quantum dot solar cells (CJQDSCs) are fabricated from as-prepared highly monodispersed lead sulfide QDs. The cells have a high power conversion of $9.05 \%$ and a short-circuit current density of $32.51 \mathrm{~mA} \mathrm{~cm}^{-2}$. A reliable and effective stratagem for fabricating high-quality lead sulfide quantum dots (QD) is explored through a "monomer" concentration-controlled experiment. Robust QDSC performances with different band gaps are demonstrated from the as-proposed synthesis and processing stratagems. Various potential CJQDSCs can be envisioned from the band edge evolution of the QDs as a function of size and ligands reported here.

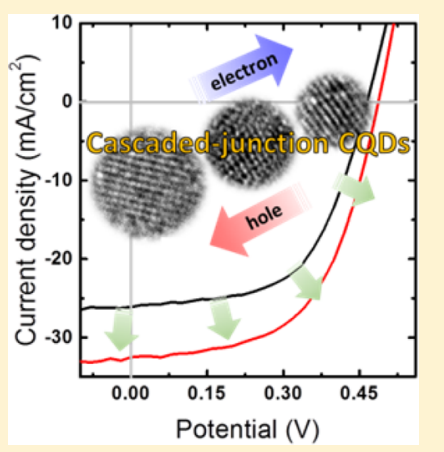

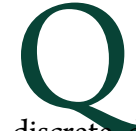
uantum dots (QDs) are well-known as infinitesimal semiconducting nanocrystals with a physical size in the range of their Bohr radius. ${ }^{1}$ Because of their discrete density of state, the band gap $\left(\varepsilon_{\text {gap }}\right)$ of QDs can be compatibly modified by manipulating their dimensions, which leads to extensive studies for optoelectronics applications. ${ }^{2}$ In particular, facile solution processability and $\varepsilon_{\text {gap }}$ customizability to the solar spectrum makes QDs one of the most promising materials for future emerging solar cells. ${ }^{3}$ Prevalent studies in QD solar cells (QDSCs), mainly concern lead sulfide (PbS) materials because of their large Bohr radius $(20 \mathrm{~nm})$ and wide band gap $\left(\varepsilon_{\text {gap }}\right)$ tuning range $(0.4-1.5 \mathrm{eV}) .{ }^{4}$ Profiting from improved process technologies, i.e. better passivation and optimized $\mathrm{p}-\mathrm{n}$ junction structure, remarkable power conversion efficiencies (PCEs) of ca. 10\% have been achieved recently. ${ }^{5,6}$ However, in spite of the demonstrated abilities and fascinating features in the QDSCs, there are still challenges which need to be addressed in terms of material quality control and device architecture design. ${ }^{3}$ For instance, a vast number of works have been performed to synthesize high-quality $\mathrm{PbS}$ QDs, ${ }^{7,8}$ but it is still a challenge to reproduce identical QDs from different batches, which hampers stable device performance. As one of the most promising QD device architectures, solar cells made from cascading various sizes of QDs have been proposed and tentatively studied. ${ }^{9-13}$ However, because of poor size control of the QDs, to date, none of the works report good PCE performance.
In this work, we elucidate an effective and reliable $\mathrm{PbS} \mathrm{QD}$ synthesis protocol for fabricating high-performance and robust QDSCs. Through the systematic adjustment of the precursor concentration, in a fixed reaction temperature and quench time, a wide range of different sizes of colloidal $\mathrm{PbS}$ QDs is produced with a narrow size distribution and high reproducibility. The effects of quantum confinement and surface functionalization for different ligands and QD size is subject to a rationalization analysis. Finally, based on the understanding gained of the optical-electrical properties of as-prepared $\mathrm{PbS} \mathrm{QDs}$, three distinct sizes of $\mathrm{PbS} \mathrm{QDs}$ are selected and fabricated into cascaded-junction solar cells (CJSC) under ambient air conditions. The device structure is illustrated in Figure 1a, which employs layers of different sizes of QDs treated with different ligands for tuning their relative band alignment and also photon energy absorption. The elaborately designed devices show impressively high PCE and short-circuit current density compared with those of previously reported devices. ${ }^{5,6}$

The assembling of CJSC requires highly monodispersed $\mathrm{PbS}$ QDs with a range of different possible sizes, ensuring small coplanar charge transport barriers and distinct size-dependent optical properties. ${ }^{2}$ QDs utilized in the light absorber layers

Received: July 25, 2016

Accepted: September 25, 2016

Published: September 28, 2016 


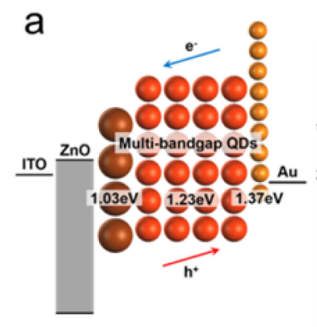

b

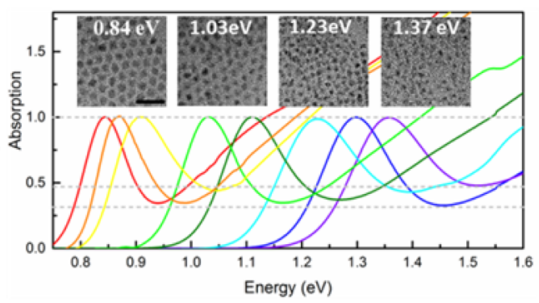

C

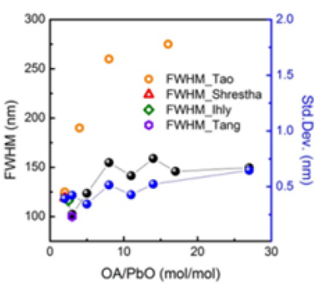

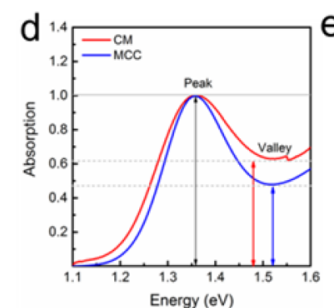
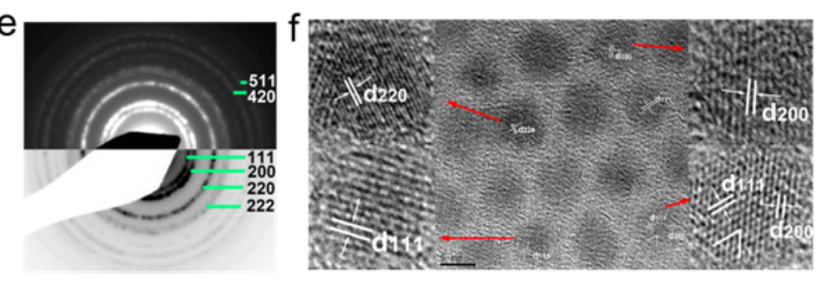

Figure 1. (a) Schematic of the proposed cascaded-junction cell with optimum combination of PbS QDs. (b) Optical absorption spectra of different sizes of $\mathrm{PbS} \mathrm{QD}$ synthesis from a series of control experiments. The values of optical $\varepsilon_{\text {gap }}$ of $\mathrm{PbS} \mathrm{QDs}$ range from 1.37 to $0.84 \mathrm{eV}$, and the corresponding mole ratio range between $\mathrm{OA}$ and $\mathrm{PbO}$ ranges from 2:1 to 27:1. The inset shows TEM images of as-prepared PbS QDs with different optical $\varepsilon_{\text {gap }}$ t the scale bar in the image is equal to $20 \mathrm{~nm}$. Horizontal short dashed lines are the reference lines for calculating peak-to-valley ratios. (c) fwhm (black) and TEM size variation results (blue) obtained from the optical absorption spectra and TEM size distribution analysis. Color symbols are previously reported values. ${ }^{19-22}$ (d) Comparison of the first exciton peak of $1.37 \mathrm{eV}$ PbS QDs synthesized from CM method (red curve) and MCC method (blue curve). Curve arrows indicate the narrowing trend between the two approaches. Vertical arrows indicate the peak-to-valley ratio between CM (1.61) and MCC (2.2) methods. (e) SAED and (f) HRTEM images of as-prepared $0.84 \mathrm{eV}$ PbS QDs. Scale bar equals $5 \mathrm{~nm}$.

shall be selected from the best performance single $\varepsilon_{\text {gap }}$ QDSC, which possess high values of PCE and quantum efficiency. Through the size and ligand engineering, the band alignments of the selected QDs shall also facilitate effective dissociation of excitons and transfer of the photogenerated charge carriers toward the corresponding cathode and anode in the QDSCs. To these ends, we first investigate a reproducible preparation approach for highly monodispersed $\mathrm{PbS}$ QDs together with optimizing single $\varepsilon_{\text {gap }}$ QDSCs, because they are critical steps toward our proposed goal.

$\mathrm{PbS}$ QDs were prepared based on a conventional "hotinjection" approach employing a Schlenk line technique, ${ }^{7}$ but our monomer concentration control (MCC) method resulted in monodispersed $\mathrm{PbS}$ QDs with high reproducibility, which is crucial for $\mathrm{QD}$ and device manufacturing. Unlike previous works, ${ }^{7,14}$ the loading of mole ratio between lead oxide $(\mathrm{PbO})$ and oleic acid (OA) were deliberately set as $\mathrm{PbO}$ to $\mathrm{OA}$ equal to $1: 2,1: 3,1: 5,1: 8,1: 11,1: 14,1: 17$, or $1: 27$. During the synthesis, other parameters that are strongly related to control the size of the QDs were fixed with $20 \mathrm{~s}$ reaction time and 130 ${ }^{\circ} \mathrm{C}$ injection temperature. The reaction time is controlled by quenching the reaction with an ice bath rather than allowing it to cool naturally. This reliable and modularized synthesis process of $\mathrm{PbS} \mathrm{QDs}$ over a wide range of targeted size is attributed to good control of the "critical size". ${ }^{5}$ When the $\mathrm{PbO}: \mathrm{OA}$ mole ratio is changed to manipulate the $\mathrm{PbS}$ monomer concentration, the size of as-prepared $\mathrm{PbS}$ QDs can be precisely controlled. A high $\mathrm{PbS}$ monomer concentration in the nucleation stage induced a small size of PbS QDs, and a low $\mathrm{PbS}$ monomer concentration induced a big size of $\mathrm{PbS}$ QDs. Our experimental results on the size controllability can be supported by the colloid growth theory: ${ }^{15}$ (i) The nanocrystal growth time is fixed to $20 \mathrm{~s}$, which means the overall QD growth time is transient, i.e. the "nucleation stage" dominates rather than the "ripening stage". 16 (ii) The OA amount is adjusted during the $\mathrm{PbS}$ QDs synthesis, which implies that the amounts of lead monomer and the sulfur monomer remain fixed but the monomer concentration is changed depending on the OA's loading. Just as in the Sugimoto model of the growth rate versus size plot, ${ }^{17}$ the high initial monomer concentration induces a bigger "critical size" and leads to a slow growth rate of the particle (all the particles smaller than the critical size have a negative growth rate). Eventually, the high initial monomer concentration results in the formation of small-sized QDs for the fixed reaction time. In a low initial monomer concentration (as the increment of OA amount), the smaller critical size induces the very fast growth rate of the particles (particles formed at the nucleation stage which are bigger than the critical size have a positive growth rate). Ultimately, low initial monomer concentrations induce the formation of large PbS QDs for the fixed reaction time.

Absorption spectroscopy analysis was carried out to evaluate the size distribution and $\varepsilon_{\text {gap }}$ variation of the as-prepared $\mathrm{PbS}$ QDs. As shown in Figure $1 \mathrm{~b}$, the $\varepsilon_{\text {gap }}$ obtained from asprepared QDs can cover from 0.84 to $1.37 \mathrm{eV}$, which encompasses the optimal energy gap for a single-junction cell (Shockley-Queisser limit, ca. $1.3 \mathrm{eV}) .^{18} \mathrm{~A}$ group of transmission electron microscopy (TEM) images (20 nm scale bar) of four different sizes of $\mathrm{PbS}$ QDs with the corresponding $\varepsilon_{\text {gap }}$ from 0.84 to $1.37 \mathrm{eV}$ are exhibited in inset image of Figure $1 \mathrm{~b}$. It can be seen that the as-prepared $\mathrm{PbS}$ QDs show a clear monodispersity among different $\varepsilon_{\text {gap }}$. The monodispersity of $\mathrm{PbS} \mathrm{QDs}$ is evaluated from a full width at half-maximum (fwhm) of the absorption spectrum and the standard deviation (SD) of the TEM size distribution. Regardless of the QD size, a sharp narrow first exciton peak is a common feature in Figure $1 \mathrm{~b}$, and the fwhm of the peaks ranges from 100 to $150 \mathrm{~nm}$ (Figure 1c). Based on the particle size TEM statistical calculations, the as-prepared $\mathrm{PbS}$ QDs possess a SD below $0.65 \mathrm{~nm}$ (detail TEM analysis can be found in Figures S1-S7 in the Supporting Information). When fwhm values from previous reports (e.g., 100-275 nm) are compared, as can be seen in 
a

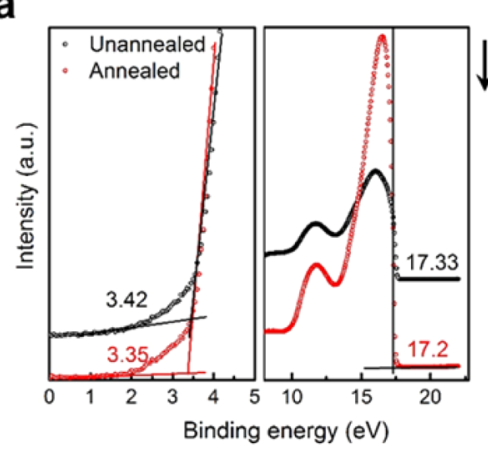

C

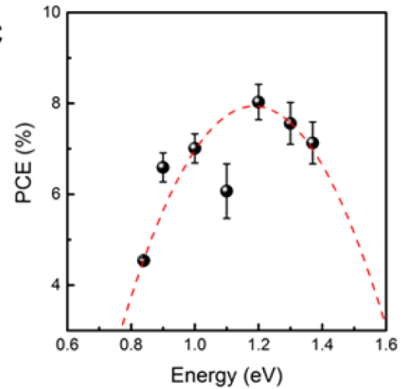

b
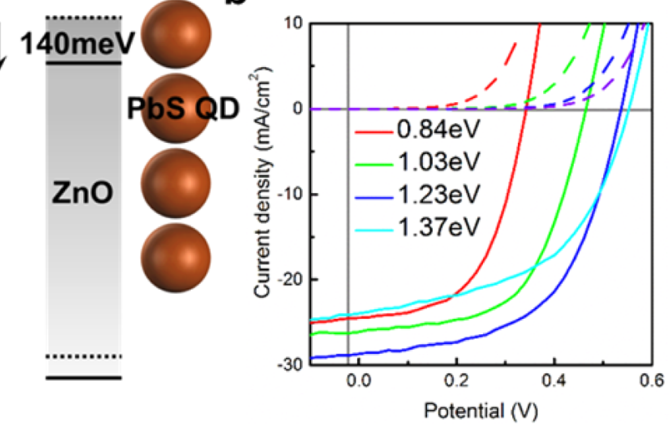

d

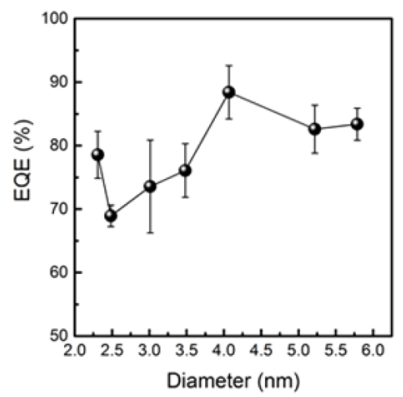

Figure 2. (a) UPS spectra of unannealed $\mathrm{ZnO}$ film (black curve) and annealed $\mathrm{ZnO}$ (red curve) film. The left panel shows magnified spectra near the Fermi edge, and the right panel shows the secondary electron cutoff region. Spectra were shifted for clarity, and the identified binding energy values are listed beside each plot. $\varepsilon_{\text {gap }}$ of annealed (bulk) and as-prepared $\mathrm{ZnO}$ NPs are employed as 3.37 and $3.45 \mathrm{eV}$, respectively. ${ }^{27}$ The band alignment schematic of annealed (solid line) and unannealed (dotted line) ZnO NP films relative to 1.3 eV PbS@TBAI at opencircuit conditions is also provided to exemplify the band edge shift. (b) Representative $J-V$ curves of QDSC with PbS QDs $\varepsilon_{\text {gap }}$ of $0.84 \mathrm{eV}$ (red curves), $1.03 \mathrm{eV}$ (green curves), $1.23 \mathrm{eV}$ (blue curves), and $1.37 \mathrm{eV}$ (light blue) under dark conditions (dashed line) and $1.5 \mathrm{AM}$ illumination (continuous line). (c) PCE values (black legend) evolution as a function of QDs optical $\varepsilon_{\text {gap }}$. The red dotted line is a Gaussian data fitting for guiding the PCE changing trend; the error bars are generated from standard deviation of parallel optimized solar cells. (d) Peak EQE values measured at $400 \mathrm{~nm}$ wavelength as a function of $\varepsilon_{\text {gap. }}$ The error bars are generated from multiple parallel solar cells measurement.

Figure 1c, our MCC method produced highly monodispersed QDs with identical or smaller fwhm values. ${ }^{14,19-22}$ It should be noted that the variation of SD (blue circles) and fwhm (black circles) as a function of QD size is also very small (as shown in Figure 1c). These experimental results on the monodispersity of QDs fit well with the theoretical simulations in Talapin et al.'s report which show that by adjusting the initial monomer concentration, a minimization of SD as a function of size is expected. ${ }^{16}$ That is, an adjustment of monomer concentration accompanied by a change in targeted QD size can bridge the gap of the "focusing", "defocusing", or "equilibrium" processes during the growth of colloidal particle, which ultimately contributes to the uniform size monodispersity. ${ }^{16}$ Two representative $\mathrm{PbS}$ QDs with $\varepsilon_{\text {gap }}=1.37$ or $0.84 \mathrm{eV}(0.84 \mathrm{eV}$ QD shown in Figure S9) were fabricated through conventional time-dependent method (CM) and MCC experiments. It can be seen from Figure 1d that the first exciton peak from the absorption spectrum shows a narrowing trend (as indicated by the arrows) between the MCC and CM. The peak-to-valley ratio is also enlarged when employing the MCC method. The peak-to-valley ratios among the different sizes of QDs extracted from the absorption spectra is within 3.5 to 2 as shown in Figure S9b.

The high crystallinity and the rock-salt cubic crystal structure of as-prepared $\mathrm{PbS}$ QDs are demonstrated from high-resolution TEM (HRTEM), selected area diffraction (SAED), and X-ray diffraction (XRD) analysis. The SAED pattern and HRTEM images in Figure 1e,f were taken from $0.84 \mathrm{eV} \mathrm{PbS}$ QDs; the other $\varepsilon_{\text {gap }} \mathrm{PbS}$ QDs microscopy studies and XRD analysis can be found in Figures S1-S8a. In Figure 1e, typical polycrystalline continuous ring SAED patterns are clearly resolved. As indexed in the diffraction pattern, the main lattice diffractions arise from $\{111\},\{200\},\{220\},\{222\},\{420\}$, and $\{511\}$ planes. An average value of the lattice constant, $a$, computed from each reflection is $5.89 \pm 0.05 \AA$, which is quite close to the reported $\mathrm{PbS}$ bulk value $5.936 \AA^{23}$ Regarding individual particles, the HRTEM study was performed to understand the microstructure of the as-prepared QDs. As shown in Figure 1f, (111), (200), and (220) planes can be readily indexed, and the lattice distances of these planes are measured to be $3.42 \pm 0.01$, $2.9 \pm 0.01$, and $2.1 \pm 0.01 \AA$, respectively, which gives a cubic $\mathrm{PbS}$ lattice constant of $a=5.89 \pm 0.02 \AA$. A cross-grating pattern was also resolved as highlighted in Figure 1f; the angle between (111) and (200) planes is measured to be $55 \pm 1^{\circ}$, which further demonstrated the $\mathrm{PbS} \mathrm{QD}$ cubic feature. Guidance for the preparation of each size of $\mathrm{PbS} \mathrm{QD}$ as a function of $\mathrm{OA}: \mathrm{PbO}$ mole ratio is also provided in Figure S8b. As can be seen from Figure S8b, the diameter of as-prepared $\mathrm{PbS}$ QDs, measured from TEM and XRD (calculated from Scherrer equation) and computed from the empirical equation, ${ }^{24}$ are found to correlate well with each other.

In our optimized cell fabrication condition, $\mathrm{ZnO}$ film, a hole block layer in QDSC, is fabricated by spin-coated $\mathrm{ZnO}$ nanoparticles (NPs) with an additional thermal annealing procedure. We attribute the benefits of the annealing to the increment of band offset $(140 \mathrm{meV})$ between $\mathrm{ZnO}$ and 
Table 1. Summary of $V_{\mathrm{oc}} J_{\mathrm{sc}}, R_{\mathrm{s}}, R_{\mathrm{sh}}$ FF, and PCE Average Values of As-Prepared Solar Cells Correlated with QD Optical $\varepsilon_{\mathrm{gap}}$

\begin{tabular}{|c|c|c|c|c|c|c|c|}
\hline type & $\varepsilon_{\text {gap }}(\mathrm{eV})$ & $V_{\mathrm{oc}}(\mathrm{V})$ & $J_{\mathrm{sc}}\left(\mathrm{mA} \mathrm{cm}^{-2}\right)$ & $R_{\mathrm{s}}\left(\Omega \mathrm{cm}^{2}\right)$ & $R_{\mathrm{sh}}\left(\Omega \mathrm{cm}^{2}\right)$ & $\mathrm{FF}$ & PCE (\%) \\
\hline QDSC & 0.84 & $\begin{array}{l}0.35 \pm 0.01 \\
\quad(0.36)\end{array}$ & $22.66 \pm 1.39(24.47)$ & $\begin{array}{l}4.54 \pm 2.79 \\
\quad(4.88)\end{array}$ & $\begin{array}{l}314.07 \pm 32.99 \\
\quad(311.79)\end{array}$ & $0.57 \pm 0.02(0.59)$ & $4.54 \pm 0.06(4.60)$ \\
\hline QDSC & 0.91 & $\begin{array}{l}0.44 \pm 0.01 \\
\quad(0.46)\end{array}$ & $27.58 \pm 1.34(29.63)$ & $\begin{array}{l}8.47 \pm 2.86 \\
\quad(7.04)\end{array}$ & $\begin{array}{l}296.19 \pm 23.99 \\
\quad(317.06)\end{array}$ & $0.54 \pm 0.01(0.55)$ & $6.59 \pm 0.32(7.06)$ \\
\hline QDSC & 1.03 & $\begin{array}{l}0.47 \pm 0.01 \\
\quad(0.48)\end{array}$ & $25.46 \pm 1.05(26.75)$ & $\begin{array}{l}3.49 \pm 1.84 \\
(6.82)\end{array}$ & $\begin{array}{l}267.64 \pm 20.00 \\
\quad(239.04)\end{array}$ & $0.59 \pm 0.02(0.6)$ & $7.01 \pm 0.32(7.28)$ \\
\hline QDSC & 1.14 & $\begin{array}{l}0.53 \pm 0.01 \\
\quad(0.54)\end{array}$ & $22.70 \pm 1.92(25.36)$ & $\begin{array}{l}8.34 \pm 1.94 \\
(8.59)\end{array}$ & $\begin{array}{l}213.28 \pm 24.86 \\
\quad(255.30)\end{array}$ & $0.50 \pm 0.02(0.53)$ & $6.07 \pm 0.60(6.89)$ \\
\hline QDSC & 1.23 & $0.54(0.54)$ & $26.77 \pm 1.77(28.86)$ & $\begin{array}{l}5.39 \pm 1.55 \\
(5.26)\end{array}$ & $\begin{array}{l}253.46 \pm 30.08 \\
\quad(264.76)\end{array}$ & $0.56 \pm 0.02(0.58)$ & $8.03 \pm 0.39(8.55)$ \\
\hline QDSC & 1.30 & $\begin{array}{l}0.58 \pm 0.02 \\
\quad(0.60)\end{array}$ & $24.48 \pm 1.15(26.39)$ & $\begin{array}{l}5.80 \pm 1.73 \\
(4.32)\end{array}$ & $\begin{array}{l}197.99 \pm 19.41 \\
\quad(198.59)\end{array}$ & $0.54 \pm 0.03(0.57)$ & $7.56 \pm 0.46(8.07)$ \\
\hline QDSC & 1.37 & $\begin{array}{l}0.55 \pm 0.01 \\
(0.56)\end{array}$ & $26.06 \pm 1.34(27.52)$ & $\begin{array}{l}9.17 \pm 2.22 \\
(7.85)\end{array}$ & $\begin{array}{l}181.40 \pm 33.87 \\
\quad(226.78)\end{array}$ & $0.49 \pm 0.02(0.51)$ & $7.13 \pm 0.46(7.63)$ \\
\hline CJQDSC & $1.03 / 1.23 / 1.37$ & $\begin{array}{l}0.50 \pm 0.02 \\
\quad(0.52)\end{array}$ & $31.24 \pm 0.92(32.51)$ & $\begin{array}{l}4.60 \pm 1.70 \\
(4.30)\end{array}$ & $\begin{array}{l}304.35 \pm 14.52 \\
\quad(318.249)\end{array}$ & $0.56 \pm 0.03(0.59)$ & $8.67 \pm 0.28(9.05)$ \\
\hline $\operatorname{QDSC}^{b}$ & 1.33 & 0.55 & 26.5 & - & - & 0.63 & 8.55 \\
\hline QDSC $^{c}$ & 1.37 & 0.64 & 22.6 & - & - & 0.73 & 10.18 \\
\hline
\end{tabular}

${ }^{a}$ Champion devices are quoted in parentheses. Single-junction QDSC results are averaged across 9 samples on 3 different substrates. CJQDSC results are averaged across 30 samples on 10 different substrates. PCE of champion devices are quoted in parentheses. ${ }^{b}$ Values are cited from ref 6 (Chuang et al.), which is the first reported high-performance PbS QDSC above 8\% PCE. ' Values are cited from ref 5 (Lan et al.), which is the world record PbS QDSC with $10.18 \%$ PCE.
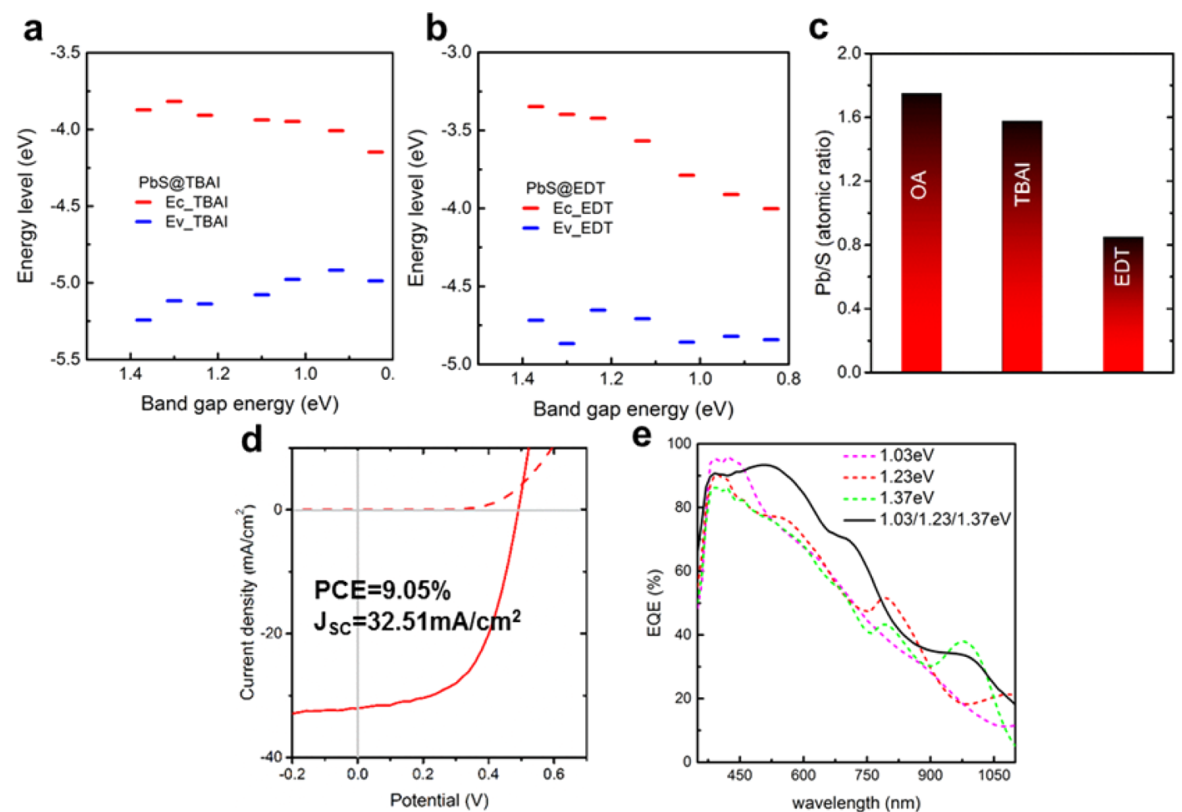

Figure 3. Size dependence of the $\varepsilon_{\text {edge }}$ energy ( $\varepsilon$ ) vs vacuum of PbS QDs capped by TBAI (PbS@TBAI) (a) and EDT (PbS@EDT) (b). (c) Quantized XPS results of the atomic ratio between $\mathrm{Pb}$ and $\mathrm{S}$ after decorating $\mathrm{PbS}$ with different ligands. (d) $J-V$ curves of a champion CJQDSC with $\mathrm{PbS}$ QDs $\varepsilon_{\text {gap }}$ of $1.03 \mathrm{eV} / 1.23 \mathrm{eV} / 1.37 \mathrm{eV}$ under dark conditions (dashed line) and 1.5 AM illumination (continuous line). Inset text highlights the champion cell PCE performance. (e) Representative EQE spectra of single $\varepsilon_{\text {gap }}$ QDSC (dashed line) and multiple $\varepsilon_{\text {gap }}$ CJQDSC (solid line). Specifications for each solar cell $\varepsilon_{\text {gap }}$ value are indicated in the image.

adjacent QD layers, which provides an energy barrier that prevents photogenerated electrons flowing back to the QD layer. Ultraviolet photoelectron spectroscopy (UPS) analysis (Figure 2a) reveals the effects of annealing on the position of the $\mathrm{ZnO}$ film band edges, and detailed discussion can be found in Figure S10 and section SVI of the Supporting Information. Figure $2 \mathrm{~b}$ exhibits four representative $J-V$ curves of asfabricated single-junction PbS QDSCs. Accompanied by changing $\varepsilon_{\text {gap }}$ from 0.84 to $1.37 \mathrm{eV}$, perceptible increments of open-circuit voltage $\left(V_{\mathrm{oc}}\right)$ can be resolved from the intersections of the abscissa with the $x$-axis. ${ }^{25}$ Figure $2 \mathrm{c}$ displays PCE values of the single-junction QDSCs fabricated from different sizes of QDs, and the highest PCE values fabricated under our optimized conditions are found from $1.23 \mathrm{eV} \mathrm{PbS}$ QDs. Essential parameters such as $V_{\mathrm{oc}} J_{\mathrm{sc}}$, series resistance $\left(R_{\mathrm{s}}\right)$, shunt resistance $\left(R_{\mathrm{sh}}\right)$, fill factor $(\mathrm{FF})$, and PCE values are extracted from the $J-V$ curves and are listed in Table 1 (The $J_{\mathrm{SC}}$ values calculated by integrating the EQE spectra with the AM 1.5 G TILT (ASTM Standard G173-03) solar spectrum are also listed in Table S1). From Table 1 it can be derived that $R_{\mathrm{sh}}$ shows a size-dependent trend which may be closely related to the size-dependent quantum confinement of the QDs. Larger $\mathrm{PbS}$ QDs (smaller $\varepsilon_{\text {gap }}$ ) show bigger $R_{\mathrm{sh}}$ values (i.e., low leakage current), which suggest weak quantum confinement which 
would induce lower leakage current or charge carrier pathways in QDs and vice versa. ${ }^{26}$ Therefore, it indicates introducing larger QDs into QDSCs may help improve $R_{\mathrm{sh}}$ and subsequently decrease leakage current. Figure $2 \mathrm{~d}$ shows the peak external quantum efficiency (EQE) as a function of $\varepsilon_{\text {gap }}$ (full EQE spectra can be found in Figures S11 and S12).

Size-dependent band edge $\left(\varepsilon_{\text {edge }}\right)$ variation of $\mathrm{PbS} \mathrm{QDs}$ stabilized with two prevailing ligands [i.e., tetrabutylammonium iodide (PbS@TBAI) and 1, 2-ethanedithiol (PbS@EDT)] are also studied by the UPS measurement. As shown in Figure 3, the conduction band edge $\left(\varepsilon_{\mathrm{C}}\right)$ and valence band edge $\left(\varepsilon_{\mathrm{V}}\right)$ evolve as a function of $\mathrm{PbS} \mathrm{QDs}$ size, exhibiting different behavior between the TBAI (Figure 3a) and EDT (Figure 3b) functionalization. In the case of TBAI-capped PbS QDs, the $\varepsilon_{\mathrm{C}}$ energy level dropped at a similar rate with the increasing of $\varepsilon_{\mathrm{V}}$ energy levels, resulting in a convergence of energy levels as a function of $\mathrm{QD}$ size. This type of $\varepsilon_{\text {edge }}$ variation (i.e., convergence) is consistent with the identical small effective mass of electrons and holes in lead salts, which induces large confinement energies split about equally between carriers. ${ }^{4}$ On the other hand, the EDT-capped PbS QDs display a trend that is different than that of TBAI-capped QDs. In Figure $3 b$, the $\varepsilon_{\mathrm{V}}$ energy levels of the EDT-treated PbS QDs only subtly change (remaining nearly steady) as the size of QDs varied, but the $\varepsilon_{\mathrm{C}}$ energy levels change dramatically. ${ }^{28}$ The different behaviors of $\varepsilon_{\text {edge }}$ energy level variation between TBAI- and EDT-treated $\mathrm{PbS}$ QDs can be attributed to the stoichiometry transformation. The atomic ratios of $\mathrm{Pb}$ and $\mathrm{S}$ analyzed by quantized $\mathrm{X}$-ray photoelectron spectroscopy (XPS) from different ligands show that the TBAI decoration brought only negligible changing of the $\mathrm{Pb} / \mathrm{S}$ ratio, but the treatment of EDT dramatically modified the $\mathrm{Pb} / \mathrm{S}$ ratio from $\mathrm{Pb}$-rich to $\mathrm{S}$-rich (Figure 3c, Figure S13, and Table S2). ${ }^{29}$ On the basis of the previously reported firstprinciples calculations, the inception of this is related to the anomalous nature of the lead chalcogenide relative to the conventional zinc-blende II-VI and III-V compounds. ${ }^{30,31}$ For instance, the $\mathrm{Pb}$ 6s band was found to lie below the top of the valence band, and the existence of an occupied $\mathrm{Pb} s$ band leads the $S \mathrm{p}$ orbitals also contributing to the conduction band..$^{30,31}$ Consequently, a strong $\mathrm{S} \mathrm{p}$ and $\mathrm{Pb}$ s coupling ( $\mathrm{L}$ point in the Brillouin zone) facilitates a "balancing act" that induces a conduction band more sensitive to the anion variation. ${ }^{30,31}$ This finding suggests a modification of stoichiometry of PbS QDs (from surface or integrity) will have a remarkable effect on the electronic structure of PbS QDs. ${ }^{32}$ Furthermore, the $\varepsilon_{\text {edge }}$ diagrams of TBAI- or EDT-functionalized QDs are actually an guidance for CJQDSC fabrication, because type II junctions can be easily formed between PbS@TBAI and PbS@EDT QDs, or even PbS@EDT QDs themselves. These CJQDSCs combine "junction", "ligand", and "QD size" effects into one case, which can promote wider photon energy absorption and higher electron-hole pair-splitting efficiency. Specifically, in this type of QDSC, photons of different energies can be absorbed preferentially in layers of different $\varepsilon_{\text {gap }}$, so the loss of electronic kinetic energy and extracted electrochemical potential can also be effectively reduced. ${ }^{25}$ Moreover, employing QDs with different $\varepsilon_{\text {gap }}$ but made of the same substance can result in the minimization of the lattice mismatch and enhancement of the built-in electric field between junctions. ${ }^{25}$

Based on the aforementioned systematic studies on the properties of QDs and QDSCs, three distinct sizes of QDs (PCE champion $1.23 \mathrm{eV}$ QDs, the Shockley-Queisser limit preferred $1.37 \mathrm{eV} \mathrm{QD}$, and high EQE $1.03 \mathrm{eV} \mathrm{QD})$ were selected for the fabrication of CJQDSCs. In consideration of size difference of the QDs, QD film thicknesses are evaluated by atomic force microscopy and scanning electron microscopy (Figures S14 and S15) before each type of solar cell fabrication. Typically, in the CJQDSC, a layer of the $1.03 \mathrm{eV}$ QD@TBAI serves as absorber and hole-extractor; 10 layers of $1.23 \mathrm{eV}$ QD act as the main light absorbing layer; and a layer of $1.37 \mathrm{eV}$ QD serves as an electron-blocking/hole-extracting layer (detailed solar cell fabrication procedures can be found in the Supporting Information). As shown in Figure $3 \mathrm{~d}$, we successfully demonstrate that the CJQDSC can generate a 9.05\% PCE performance, which is higher than any of its constituent QDSCs, with a particularly outstanding high $J_{\mathrm{sc}}$ and high $R_{\mathrm{sh}}$. Figure $3 \mathrm{e}$ shows EQE performances of the typical CJQDSC and its component individual solar cells. The highest EQE comes from the $1.03 \mathrm{eV}$ QDSC, but the CJQDSC shows the best spectrum coverage and $\mathrm{EQE}$ performance higher than that of the 1.23 and $1.37 \mathrm{eV}$ QDSCs. As a result of the improved optical absorption (Figure S16), the $J_{\text {sc }}$ values of CJQDSCs can be increased up to $32.51 \mathrm{~mA} \mathrm{~cm}{ }^{-2}$, which is the highest value among any reported values in PbS QDSCs. ${ }^{5,6}$ The $J_{\mathrm{SC}}$ value calculated by integrating the EQE spectra with the AM1.5G solar spectrum for CJQDSC is $30.11 \mathrm{~mA} / \mathrm{cm}^{2}(28.01 \pm 2.10$ $\mathrm{mA} / \mathrm{cm}^{2}$ ), which shows a good agreement with the measured $J_{S C}$. Furthermore, the PbS CJQDSC shows a robust stability; it can be fabricated in air and retains a high PCE performance under ambient air conditions for more than 20 weeks (Figure S17).

Comparisons between our single $\varepsilon_{\text {gap }}$ cells, CJQDSCs, and the worldwide leading results are listed in Table 1 . In spite of the promising performance of our CJQDSCs, there are still a vast number of parameters which can be optimized to reach the leading devices. For example, our FF and $V_{\text {oc }}$ values are smaller than the worldwide best cell, which may result from a poor packing density and interface defects. Ongoing work such as improving QD layer packing densities, reducing multiple junction interface potential barriers, and tackling the $V_{\text {oc }}$ deficiency ${ }^{32}$ are being carried out in our group.

In summary, highly monodispersed $\mathrm{PbS}$ QDs are fabricated from the MCC method. Experimentally, the size deviation of the as-prepared QDs is demonstrated to be smaller than that of the CM approach. An annealed zinc oxide layer is found to be of benefit for ensuring the robust performance of QDSCs, and $1.23 \mathrm{eV} \mathrm{PbS}$ QDs show the best single-junction solar cell performance in our optimized conditions. From UPS analysis, distinct $\varepsilon_{\text {edge }}$ shifting trends are discovered between TBAI- and EDT-treated $\mathrm{PbS} \mathrm{QDs}$, which are attributed to the stoichiometry variation during the ligand exchange. In view of the appropriate band alignments among various $\varepsilon_{\text {gap }}$ QDs, the CJQDSC prototype is proposed and successfully constructed. In the end, we demonstrate the credibility of these multiple $\varepsilon_{\text {gap }}$ homojunction solar cells by its outstanding photovoltaic performance. We believe the MCC QD synthesis protocol and CJQDSC model can be a promising candidate encouraging future high-performance QDSC studies.

\section{ASSOCIATED CONTENT}

\section{S Supporting Information}

The Supporting Information is available free of charge on the ACS Publications website at DOI: 10.1021/acsenergylett.6b00294. 
Experimental sections including the preparation methods of materials and devices; material and device characterizations such as microscopes, diffractions, ultraviolet photoelectron spectroscopy, X-ray photoelectron spectroscopy, and external quantum efficiencies (PDF)

\section{AUTHOR INFORMATION}

\section{Corresponding Authors}

*E-mail: junginn.sohn@eng.ox.ac.uk.

*E-mail: seungnam.cha@eng.ox.ac.uk.

\section{Notes}

The authors declare no competing financial interest.

\section{ACKNOWLEDGMENTS}

The research leading to these results has received funding from the European Research Council under ERC Grant Agreement 340538. The authors also thank the National Research Foundation (NRF) of Korea (2015M2A2A6A02045252) and the framework of Research and Development Program of the Korea Institute of Energy Research (KIER, B6-2498) for financial support.

\section{REFERENCES}

(1) Brus, L. E. Electron-Electron and Electron-Hole Interactions in Small Semiconductor Crystallites: the Size Dependence of the Lowest Excited Electronic State. J. Chem. Phys. 1984, 80, 4403-4409.

(2) Konstantatos, G; Sargent, E. H. Colloidal Quantum Dot Optoelectronics and Photovoltaics; Cambridge University Press: Cambridge, U.K., 2013.

(3) Kim, M. R; Ma, D. Quantum-Dot-Based Solar Cells: Recent Advances, Strategies, and Challenges. J. Phys. Chem. Lett. 2015, 6, 8599.

(4) Wise, F. W. Lead Salt Quantum Dots: the Limit of Strong Quantum Confinement. Acc. Chem. Res. 2000, 33, 773-780.

(5) Lan, X.; Voznyy, O.; Kiani, A.; García de Arquer, F. P.; Abbas, A. S.; Kim, G.-H.; Liu, M.; Yang, Z.; Walters, G.; Xu, J.; et al. Passivation Using Molecular Halides Increases Quantum Dot Solar Cell Performance. Adv. Mater. 2016, 28, 299-304.

(6) Chuang, C.-H. M.; Brown, P. R.; Bulović, V.; Bawendi, M. G. Improved Performance and Stability in Quantum Dot Solar Cells through Band Alignment Engineering. Nat. Mater. 2014, 13, 796-801.

(7) Hines, M. A.; Scholes, G. D. Colloidal PbS Nanocrystals with Size-Tunable Near-Infrared Emission: Observation of Post-Synthesis Self-Narrowing of the Particle Size Distribution. Adv. Mater. 2003, 15, $1844-1849$.

(8) Cademartiri, L.; Bertolotti, J.; Sapienza, R.; Wiersma, D. S.; von Freymann, G.; Ozin, G. A. Multigram Scale, Solventless, and DiffusionControlled Route to Highly Monodisperse PbS Nanocrystals. J. Phys. Chem. B 2006, 110, 671-673.

(9) Neo, D. C. J.; Stranks, S. D.; Eperon, G. E.; Snaith, H. J.; Assender, H. E.; Watt, A. A. R. Quantum Funneling in Blended MultiBand Gap Core/Shell Colloidal Quantum Dot Solar Cells. Appl. Phys. Lett. 2015, 107, 103902.

(10) Choi, J. J.; Wenger, W. N.; Hoffman, R. S.; Lim, Y.-F.; Luria, J.; Jasieniak, J.; Marohn, J. A.; Hanrath, T. Solution-Processed Nanocrystal Quantum Dot Tandem Solar Cells. Adv. Mater. 2011, 23, 3144-3148.

(11) Santra, P. K.; Kamat, P. V. Tandem-Layered Quantum Dot Solar Cells: Tuning the Photovoltaic Response with Luminescent Ternary Cadmium Chalcogenides. J. Am. Chem. Soc. 2013, 135, 877-885.

(12) Semonin, O. E.; Luther, J. M.; Beard, M. C. Quantum Dots for Next-Generation Photovoltaics. Mater. Today 2012, 15, 508-515.

(13) Wang, X.; Koleilat, G. I.; Tang, J.; Liu, H.; Kramer, I. J.; Debnath, R.; Brzozowski, L.; Barkhouse, D. A. R.; Levina, L.; Hoogland, S.; et al. Tandem Colloidal Quantum Dot Solar Cells
Employing a Graded Recombination Layer. Nat. Photonics 2011, 5, $480-484$.

(14) Zhang, J.; Crisp, R. W.; Gao, J.; Kroupa, D. M.; Beard, M. C.; Luther, J. M. Synthetic Conditions for High-Accuracy Size Control of PbS Quantum Dots. J. Phys. Chem. Lett. 2015, 6, 1830-1833.

(15) Peng, X.; Wickham, J.; Alivisatos, A. P. Kinetics of II-VI and IIIV Colloidal Semiconductor Nanocrystal Growth: "Focusing" of Size Distributions. J. Am. Chem. Soc. 1998, 120, 5343-5344.

(16) Talapin, D. V.; Rogach, A. L.; Haase, M.; Weller, H. Evolution of an Ensemble of Nanoparticles in a Colloidal Solution: Theoretical Study. J. Phys. Chem. B 2001, 105, 12278-12285.

(17) Sugimoto, T. Preparation of Monodispersed Colloidal Particles. Adv. Colloid Interface Sci. 1987, 28, 65-108.

(18) Shockley, W.; Queisser, H. J. Detailed Balance Limit of Efficiency of p-n Junction Solar Cells. J. Appl. Phys. 1961, 32, 510519.

(19) Ihly, R.; Tolentino, J.; Liu, Y.; Gibbs, M.; Law, M. The Photothermal Stability of PbS Quantum Dot Solids. ACS Nano 2011, $5,8175-8186$.

(20) Shrestha, A.; Jin, B.; Kee, T. W.; Qiao, S. Z.; Dai, S. A Robust Strategy for "Living" Growth of Lead Sulfide Quantum Dots. ChemNanoMat 2016, 2, 49-53.

(21) Tao, L.; Xiong, Y.; Liu, H.; Shen, W. High Performance PbS Quantum Dot Sensitized Solar Cells via Electric Field Assisted in situ Chemical Deposition on Modulated $\mathrm{TiO}_{2}$ Nanotube Arrays. Nanoscale 2014, 6, 931-938.

(22) Tang, J.; Brzozowski, L.; Barkhouse, D. A. R.; Wang, X.; Debnath, R.; Wolowiec, R.; Palmiano, E.; Levina, L.; PattantyusAbraham, A. G.; Jamakosmanovic, D.; et al. Quantum Dot Photovoltaics in the Extreme Quantum Confinement Regime: The SurfaceChemical Origins of Exceptional Air- and Light-Stability. ACS Nano 2010, 4, 869-878.

(23) Madelung, O.; Rössler, U.; Schulz, M. Non-Tetrahedrally Bonded Elements and Binary Compounds I: Lead sulfide (PbS) crystal structure, lattice parameters, thermal expansion; Springer: Berlin, 1998.

(24) Moreels, I.; Lambert, K.; Smeets, D.; De Muynck, D.; Nollet, T.; Martins, J. C.; Vanhaecke, F.; Vantomme, A.; Delerue, C.; Allan, G.; et al. Size-Dependent Optical Properties of Colloidal PbS Quantum Dots. ACS Nano 2009, 3, 3023-3030.

(25) Nelson, J. The Physics of Solar Cells; Imperial College Press: London, 2003.

(26) Yoon, W.; Boercker, J. E.; Lumb, M. P.; Placencia, D.; Foos, E. E.; Tischler, J. G. Enhanced Open-Circuit Voltage of PbS Nanocrystal Quantum Dot Solar Cells. Sci. Rep. 2013, 3, 2225.

(27) Masuda, Y.; Yamagishi, M.; Seo, W. S.; Koumoto, K. Photoluminescence from $\mathrm{ZnO}$ Nanoparticles Embedded in an Amorphous Matrix. Cryst. Growth Des. 2008, 8, 1503-1508.

(28) Jasieniak, J.; Califano, M.; Watkins, S. E. Size-Dependent Valence and Conduction Band-Edge Energies of Semiconductor Nanocrystals. ACS Nano 2011, 5, 5888-5902.

(29) Kim, D.; Kim, D.-H.; Lee, J.-H.; Grossman, J. C. Impact of Stoichiometry on the Electronic Structure of PbS Quantum Dots. Phys. Rev. Lett. 2013, 110, 196802.

(30) Hou, B.; Parker, D.; Kissling, G. P.; Jones, J. A.; Cherns, D.; Fermín, D. J. Structure and Band Edge Energy of Highly Luminescent $\mathrm{CdSe}_{1-\mathrm{x}} \mathrm{Te}_{\mathrm{x}}$ Alloyed Quantum Dots. J. Phys. Chem. C 2013, 117, 6814-6820

(31) Wei, S.-H.; Zunger, A. Electronic and structural anomalies in lead chalcogenides. Phys. Rev. B: Condens. Matter Mater. Phys. 1997, 55, 13605-13610.

(32) Chuang, C.-H. M.; Maurano, A.; Brandt, R. E.; Hwang, G. W.; Jean, J.; Buonassisi, T.; Bulović, V.; Bawendi, M. G. Open-Circuit Voltage Deficit, Radiative Sub-Bandgap States, and Prospects in Quantum Dot Solar Cells. Nano Lett. 2015, 15, 3286-3294. 